\title{
Deep-Inspiration Breath-Hold PET/CT of Lung Cancer: Maximum Standardized Uptake Value Analysis of 108 Patients
}

\author{
Tsuyoshi Kawano ${ }^{1}$, Eiji Ohtake ${ }^{1}$, and Tomio Inoue ${ }^{2}$ \\ ${ }^{I}$ Division of Nuclear Medicine, Kanagawa Cancer Center, Yokohama, Japan; and ${ }^{2}$ Department of Radiology, Yokohama City University \\ School of Medicine, Yokohama, Japan
}

Our aim was to compare the maximum standardized uptake value (SUVmax) between breath-hold (BH) PET/CT and freebreathing (FB) PET/CT. Methods: The features of phantom data were analyzed, after which a clinical study was performed. A total of 108 consecutive patients with lung cancer were examined using lutetium oxyorthosilicate (LSO)-based PET/CT. The patients were instructed to breathe freely during FB PET/CT. In $\mathrm{BH} \mathrm{PET} / \mathrm{CT}$, the patients were instructed to hold their breath in the maximal inspiration position during the scout scan, for $10 \mathrm{~s}$ of the CT scan, and for as long as possible during the PET scan. $\mathrm{BH}$ time was recorded using a respiratory monitoring device. The $\% \mathrm{BH}$-index was defined as the percentage difference between SUVmax of FB PET and that of BH PET. Statistical analyses were performed using the following factors: \%BH-index, age, body mass index, ${ }^{18} \mathrm{~F}-\mathrm{FDG}$ dosage, blood glucose, $\mathrm{BH}$ time, lesion size, and location. Results: The highest \% $\mathrm{BH}$-index was 223.2. $\% \mathrm{BH}$-index in the lower lung area was significantly higher than that in the upper lung area (51.8 \pm 49.5 vs. $16.9 \pm$ 25.6, respectively). Lesion volume and maximum diameter in the high-\%BH-index group were significantly lower than those in the low-\%BH-index group, with the use of a \% $\mathrm{BH}$-index cutoff value of 37.I. Conclusion: SUVmax of FB PET should not be taken as accurate, especially in the lower lung area and for small pulmonary lesions. BH PET/CT is expected to enable precise measurement of SUVmax and is thus recommended as part of the standard protocol for lung cancer.

Key Words: deep-inspiration breath-hold; PET/CT; maximum SUV; lutetium oxyorthosilicate; lung cancer

J Nucl Med 2008; 49:1223-1231

DOI: 10.2967/jnumed.107.049296

$\mathbf{W}_{\text {not }}$ hole-body ${ }^{18} \mathrm{~F}$-FDG PET is an important imaging method in modern oncology for the diagnosis of disease in many different malignant tumors. Combined PET/CT systems enable the simultaneous acquisition of morphologic and

Received Nov. 26, 2007; revision accepted Apr. 24, 2008.

For correspondence or reprints contact: Tsuyoshi Kawano, Division of Nuclear Medicine, Kanagawa Cancer Center Nakao 1-1-2, Asahi-ku, Yokohama, 241-0815, Japan.

E-mail: kawanotsuyoshi@hotmail.com

COPYRIGHT $\odot 2008$ by the Society of Nuclear Medicine, Inc. functional information. Fusion PET/CT images have affected the diagnosis of disease; however, image misregistration can occur in fusion PET/CT, particularly in the chest and abdomen, because of motion artifacts caused by respiration. $\mathrm{CT}$ is fast and enables flexible imaging protocols; many reports describe imaging protocols of the CT component of PET/CT (1-6), but a perfect match between PET and CT is impossible. The standard imaging protocol of the CT component of PET/CT is free breathing (FB) or a breathhold $(\mathrm{BH})$ in the normal expiratory position. However, the CT component of PET/CT during shallow breathing is reported to be inadequate for lung cancer imaging (7), and chest CT during deep inspiration is recommended for the thorough evaluation of patients for whom the detection of small pulmonary nodules is necessary $(8,9)$. The PET component of PET/CT takes several minutes per bed position, and the resultant image comprises images averaged over many breathing cycles. Changes in the mean and maximum standardized uptake value (SUVmax) and mislocalization of lesions have been reported previously during normal respiratory movement $(3,4,10)$. Evaluation of changes in SUVmax during respiratory movement is important because SUVmax is widely used in differentiating benign from malignant tumors (11), in determining the threshold levels of the gross tumor volume and tumor contour $(12,13)$, and in monitoring of chemoradiation therapy.

The methodology of 4-dimensional PET/CT protocols has been previously reported (14-16), but the consensus on clinical protocols for respiratory-gated PET is not yet clearly defined. The examination is clinically impractical because of patients' breathing irregularities and lengthy acquisition and postprocessing times.

Recent articles refer to the methodology involved in acquiring BH PET/CT data at end inspiration; the practice of deep-inspiration BH PET/CT has been reported as feasible in the clinical setting $(17,18)$. The use of a lutetium oxyorthosilicate (LSO) PET detector and 3-dimensional acquisition technology may enable a reduction in imaging times (19). As the previously described method $(17,18)$ could not be implemented on our PET/CT scanner, the present study proposes an original BH PET/CT technique. 
The features of phantom data were analyzed, after which a clinical study was performed.

The purpose of the present study was to compare the SUVmax of BH (SUVmaxBH) PET/CT with that of conventional FB (SUVmaxFB) PET/CT in 108 cases of lung cancer.

\section{MATERIALS AND METHODS}

\section{Phantom Study}

We used an International Electrotechnical Commission (IEC) body phantom set corresponding to the NU 2-2001 standard published by National Electrical Manufacturers Association (NEMA) (Fig. 1). The set consisted of a torso cavity, a removable lung insert, and 6 spheres (inner diameter, 10, 13, 17, 22, 28, and $37 \mathrm{~mm}$ ) that can be filled as required. The torso cavity was filled with water, and the 6 spheres were filled with an ${ }^{18}$ F-FDG solution in the same concentrations of radioactivity, with the concentrations varying as follows: 53, 13, and $7 \mathrm{kBq} / \mathrm{mL}$. The PET/CT scan was performed using a single bed position. CT was used for CT-based attenuation correction. PET emission data were acquired in consecutive 1-s time frames for $15 \mathrm{~min}$ using the list-mode dynamic collection method. A total of 5 image frames $(n=5)$ were extracted from each of the 3-min, 1-min, 30-s, and 15-s acquisition times using the raw datasets from the 15-min scan. The same clinical reconstruction parameters as those described below were used for image reconstruction. Regions of interest (ROIs) were placed around each sphere, and the maximum value in the ROI was defined as the SUVmax. The means and SDs of SUVmax for the 5 images were calculated for all spheres in the various ${ }^{18} \mathrm{~F}-\mathrm{FDG}$ radioactivity concentrations and the various acquisition times. The coefficient of variation $(\mathrm{CV})$ was defined as follows:

$$
\mathrm{CV}=\mathrm{SD} / \text { mean } \times 100 .
$$

The percentage differences between the mean SUVmax of the 3-min PET scan (SUVmax, 3 min) and the mean SUVmax of the

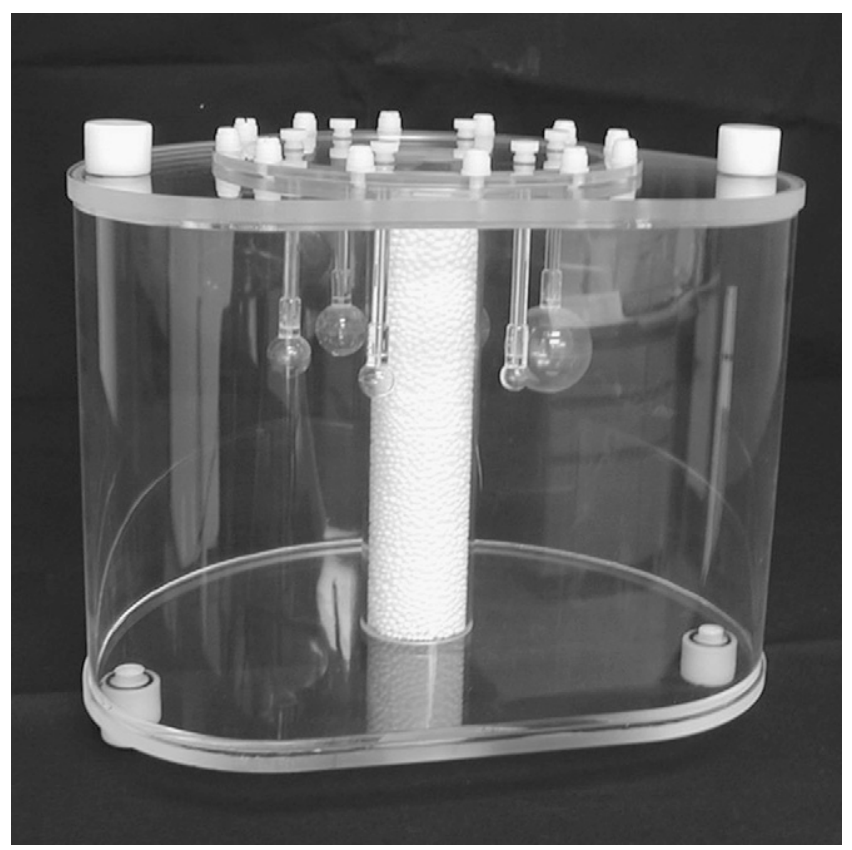

FIGURE 1. NEMA/IEC 2001 PET body phantom. 1-min, 30-s, and 15-s PET scans (SUVmax, 1 min; SUVmax, $30 \mathrm{~s}$; and SUVmax, $15 \mathrm{~s}$, respectively) were defined as follows:

$$
\begin{aligned}
\% \text { Dif-index }= & (\text { SUVmax } 1 \mathrm{~min}, 30 \mathrm{~s}, 15 \mathrm{~s}- \\
& \text { SUVmax } 3 \min ) / \text { SUVmax } 3 \min \times 100 .
\end{aligned}
$$

\section{Clinical Study}

Patients. From August 2006 to October 2007, we examined consecutive patients with a primary lesion of maximum diameter ranging from 10 to $40 \mathrm{~mm}$ for staging of lung cancer using clinical whole-body PET/CT. The protocol of this study was approved by the institutional review board, and informed consent was obtained from each patient. Diagnosis of the primary lesion was confirmed histopathologically for all patients. None of the patients had a history of chest surgical treatment. Patients with obvious hilar or pleural invasions were excluded from the study. Patients with lung tumors that showed low ${ }^{18} \mathrm{~F}-\mathrm{FDG}$ accumulation, as mentioned below, were excluded from the study. Patients who coughed, yawned, or spoke during the procedure; were deaf; or had dementia were excluded from the study. Thus, although 141 patients originally were approached to participate in this study, 33 patients $(23 \%)$ were subsequently excluded. Among the reasons for exclusion, the most frequent reason was a short $\mathrm{BH}$ (lasting fewer than $29 \mathrm{~s}$ ). Other patients were excluded for being uncooperative or because of an obvious mismatch between BH CT and BH PET. Consequently, 108 patients were evaluated in this study. Patient characteristics are summarized in Table 1.

\section{Respiratory Monitoring Device}

A respiratory gating system (AZ-733V; Anzai Medical Co. Ltd.), comprising the following components, was used for all patients. A respiratory sensor (an elasticized belt around the patient's abdomen) provides respiratory information by detecting changes in the patient's abdominal pressure. A sensor port amplifies and transmits analog signals, a wave deck receives and digitizes the signals to be sent to a personal computer, and an outputting gate signals the diagnostic imaging equipment or therapeutic irradiation equipment. Specific applications such as patient files, management of respiratory waves, setting of gate signals, and display of the respiratory waveform are installed on a personal computer. Management of the respiratory wave consists of acquisition, realtime display, storage, and reference.

\section{Data Acquisition}

All PET/CT studies were performed using an LSO-based wholebody PET/CT scanner (Biograph 16 HI-REZ; Siemens). The PET component of PET/CT has no septa, thus allowing 3-dimensionalonly acquisitions with a field of view (FOV) of $585 \mathrm{~mm}$ in the

\section{TABLE 1}

Patient Characteristics

\begin{tabular}{lcc}
\hline Characteristic & Mean \pm SD & Range \\
\hline Patient age $(\mathrm{y})$ & $66.6 \pm 9.7$ & $37-83$ \\
Weight $(\mathrm{kg})$ & $56.4 \pm 10.0$ & $36-84$ \\
Height $(\mathrm{cm})$ & $159.5 \pm 8.3$ & $141-174$ \\
BMl & $22.1 \pm 3.0$ & $15.8-30.9$
\end{tabular}

The study comprised 62 men and 46 women. 
transaxial direction and $162 \mathrm{~mm}$ in the axial direction. The intrinsic resolution is 4.2-mm full width at half maximum. The CT component of PET/CT corresponds to a 16-slice multi-detector-row spiral CT scanner (Sensation 16; Siemens) with a transverse FOV of 500 $\mathrm{mm}$ and an inplane spatial resolution greater than $1 \mathrm{~mm}$. CT scan parameters consisted of the following: collimation, $16 \times 1.5 \mathrm{~mm}$; gantry rotation time, $500 \mathrm{~ms}$; tube voltage, $120 \mathrm{kV}$; effective tube current, $50 \mathrm{mAs}$, with online tube current modulation (CARE Dose4D; Siemens); and table feed, $18 \mathrm{~mm}$ /rotation.

${ }^{18}$ F-FDG (FDGscan Injectable; Nihon Medi-Physics Co. Ltd.) was purchased via a delivery system. ${ }^{18}$ F-FDG dose per body weight cannot be determined under this delivery system because the delivery of ${ }^{18} \mathrm{~F}$-FDG occurs at 3 set times a day and there is decay from calibration time. All patients fasted for at least $6 \mathrm{~h}$ before PET/CT. Sixty minutes after intravenous administration of ${ }^{18} \mathrm{~F}-\mathrm{FDG}$ (mean dosage, $4.64 \mathrm{MBq} / \mathrm{kg}$; range, 2.17-9.53 MBq/kg), all patients were positioned supine on the imaging table with their arms by their sides and were instructed to breathe freely. Wholebody PET/CT was acquired for each patient from the top of the skull to the middle of the thigh, with $3 \mathrm{~min}$ per bed position. The conventional whole-body PET/CT scan was then evaluated to determine whether the pulmonary lesion was visible on the PET images. Patients with visible ${ }^{18}$ F-FDG accumulation in target pulmonary lesions on the whole-body PET scan participated in the present study; patients with pulmonary lesions visualized at either SUVmax of less than 2.0 or ${ }^{18} \mathrm{~F}$-FDG accumulation of less than $5 \mathrm{kBq} / \mathrm{mL}$ measured on whole-body PET were excluded.

Both FB PET/CT and BH PET/CT were performed as additional scans $110 \mathrm{~min}$ after intravenous administration of ${ }^{18} \mathrm{~F}-\mathrm{FDG}$. A conventional FB PET/CT scan with a single bed position was performed with normal respiration during an initial scout scan, CT, and PET. An initial scout scan was performed to determine whether the imaging field included a target pulmonary lesion, after which a 10-s chest CT scan was performed. PET emission data for a single bed position were acquired for at least $3 \mathrm{~min}$ using the list-mode dynamic collection method. Three-minute image frames were extracted from the raw sinogram datasets after FB PET.

$\mathrm{BH}$ PET/CT was then performed for a single bed position with the patient instructed to hold his or her breath in maximal inspiratory position during both the initial scout and the 10-s CT scans; during the PET scan, patients were asked to hold their breath in maximal inspiratory position motionlessly, as long as possible, with the respiratory monitoring device fixed. After an initial scout scan was taken to determine whether the imaging field included the target pulmonary lesion, a chest CT scan of approximately $10 \mathrm{~s}$ was performed. PET emission data for a single bed position were acquired in consecutive 1-s time frames using the list-mode dynamic collection method, until the patient could no longer hold his or her breath. The time from the beginning to the end of steady motionless $\mathrm{BH}$ was recorded by referring to the realtime display of the respiratory monitoring device. Image frames corresponding to the recorded $\mathrm{BH}$ time were extracted from the raw sinogram datasets after dynamic BH PET. Datasets from early and late periods of recorded $\mathrm{BH}$ time were not used (Fig. 2).

\section{Image Reconstruction and Analysis}

CT images were used for anatomic landmarking. CT-based attenuation correction used 700-mm extended FOV technology (Auto AC; Siemens). The same clinical reconstruction parameters were used for both the FB PET and BH PET images. All PET images were reconstructed using iterative algorithms (Fourier rebinning
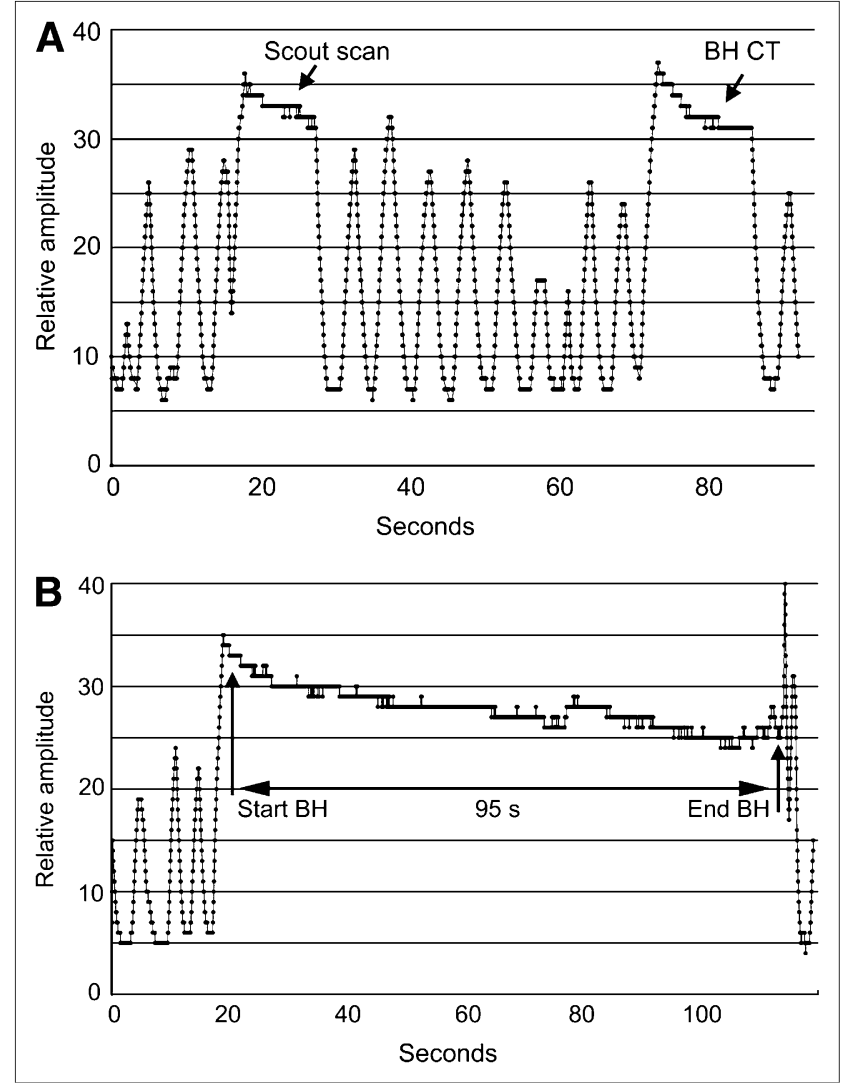

FIGURE 2. (A) Breathing signal of patient during scout scan and $\mathrm{BH}$ CT. Patient was instructed to hold breath in maximal inspiratory position during scout scan and for $10 \mathrm{~s}$ during $\mathrm{BH}$ CT scan. (B) Breathing signal during BH PET scan. Patient was instructed to hold breath in maximal inspiratory position for as long as possible during PET scan. Time from beginning to end of steady motionless $\mathrm{BH}$ was recorded as $95 \mathrm{~s}$.

plus attenuation-weighted ordered-subset expectation maximization, 2 iterations, 8 subsets, 5-mm gaussian filter) with CT-based attenuation correction. The data were reconstructed with a $168 \times 168$ matrix and 2-mm slice thickness. All PET and CT images were transformed to a Leonardo workstation (e-soft; Siemens), from which fusion PET/CT images were constructed.

ROIs were placed around the pulmonary lesions, and the maximum value in the ROI was defined as SUVmax. SUVmax was obtained for all lesions revealed on FB PET and BH PET. If multiple lesions were detected, the lesion with the highest SUVmax was used. The maximum diameters of all lesions were measured from the transaxial BH CT images or multiplanar reconstructed BH CT images. Lesion volumes were obtained by multiplying the area with maximum diameter on the transaxial BH CT image by the $z$-axis length on the BH CT image.

\section{Data Analysis and Statistical Evaluation}

The percentage difference between SUVmaxFB PET and SUVmaxBH PET was defined as follows:

$$
\begin{aligned}
\% \mathrm{BH} \text {-index }= & (\mathrm{SUVmaxBH}-\mathrm{SUVmaxFB}) / \\
& \mathrm{SUVmaxFB} \times 100 .
\end{aligned}
$$

A count recovery coefficient was not applied in the present SUV analysis. 
Analyses were performed using statistical analysis software (StatView [Windows; Microsoft]; SAS Institute Inc.). The nonparametric Mann-Whitney $U$ test was used to evaluate differences in the following factors: patient age, body mass index (BMI), ${ }^{18} \mathrm{~F}-$ FDG dosage, $\mathrm{BH}$ time, lesion maximum diameter and \%BH-index for each group (upper lung area, segments S1-S6; lower lung area, segments S7-S10; upper lobe, segments S1-S3; and middle-tolower lobe, segments S4-S10), left versus right lung, and sex. Differences in lesion volume and maximum diameter in the high$\% \mathrm{BH}$-index group and the low-\% $\mathrm{BH}$-index group were evaluated using a nonparametric Mann-Whitney $U$ test. Correlations among $\%$ BH-index, patient age, BMI, ${ }^{18} \mathrm{~F}-\mathrm{FDG}$ dosage, blood glucose level, BH time, lesion volume, and maximum diameter were assessed using an analysis of correlation coefficients. The correlation between SUVmaxFB and SUVmaxBH was also assessed.

\section{RESULTS}

\section{Phantom Study}

The results of the phantom study are shown in Tables $2-4$. The degree of activity underestimation was higher in smaller spheres, and the degree of activity overestimation was higher in larger spheres, increasing with shorter acquisition times in both cases.

\section{Clinical Study}

Data for 108 patients with 108 primary pulmonary lesions were evaluated. The mean radiation effective doses that patients received from whole-body PET/CT, FB CT, and BH CT were 7.66, 1.30, and $1.16 \mathrm{mSv}$, respectively. All of the pulmonary lesions were clearly visualized in FB PET and BH PET images. The means and SDs for SUVmaxFB and SUVmaxBH were $8.06 \pm 5.15$ and $9.78 \pm 5.76$, respectively. The mean and SD of the maximum diameters of the lesions was $2.52 \pm 0.73 \mathrm{~cm}$, ranging from 1.2 to 4.0 $\mathrm{cm}$. The mean and SD of lesion volume was $14.43 \pm 12.33$ $\mathrm{cm}^{3}$, ranging from 1.1 to $65.1 \mathrm{~cm}^{3}$. The mean and SD BH time was $54.2 \pm 20.8 \mathrm{~s}$, ranging from 30 to $125 \mathrm{~s}$. The mean and SD \%BH-index was $26.6 \pm 37.2$, ranging from -10.1 to 223.2 .

We assigned the \%BH-index to 4 groups: upper lung area, lower lung area, upper lobe, and middle-to-lower lobe. For the upper lung area $(n=78)$ and lower lung area $(n=$ 30 ), the $\% \mathrm{BH}$-index in the lower lung area was significantly higher than that in the upper lung area $(P<0.0001)$ (Table $5)$. For the upper lobe $(n=50)$ and middle-to-lower lobe $(n=58)$, the \%BH-index in the middle-to-lower lobe was significantly higher than that in the upper lobe $(P<$ 0.0001) (Table 6). No significant difference was observed in the \% BH-index between the bilateral lungs or sex.

Lesion volume and maximum diameter were assigned to 2 groups, the high-\%BH-index group $(n=24)$ and the low-\%BH-index group $(n=84)$, using a $\%$ BH-index cutoff value of 37.1. Lesion volume and maximum diameter in the high-\%BH-index group were significantly lower than those in the low-\%BH-index group $(P=0.0019$ and $P=$ 0.0032 , respectively) (Table 7).

A weak inverse relationship was observed between patient age and $\mathrm{BH}$ time $(r=-0.356, P=0.0001)$. A weak inverse relationship was also observed between \% BH-index and lesion maximum diameter $(r=-0.229, P=0.0171)$ (Fig. 3). A significant relationship was observed between SUVmaxFB and SUVmaxBH $(r=0.937, P<0.0001)$ (Fig. 4); however, the factors of patient age, BMI, ${ }^{18} \mathrm{~F}-\mathrm{FDG}$ dosage, blood glucose level, and $\mathrm{BH}$ time showed no relationship with \%BH-index.

TABLE 2

Results of Phantom Study for an ${ }^{18} \mathrm{~F}-\mathrm{FDG}$ Concentration of $53 \mathrm{kBq} / \mathrm{mL}(n=5)$

\begin{tabular}{|c|c|c|c|c|c|c|c|}
\hline \multirow[b]{2}{*}{ Acquisition time } & \multirow[b]{2}{*}{ Parameter } & \multicolumn{6}{|c|}{ Sphere size (mm) } \\
\hline & & 10 & 13 & 17 & 22 & 28 & 37 \\
\hline $15 \min$ & & 25,405 & 40,098 & 50,275 & 52,466 & 53,638 & 53,692 \\
\hline \multirow[t]{3}{*}{$3 \mathrm{~min}$} & Mean (Bq/mL) & 24,690 & 40,029 & 50,169 & 52,657 & 54,687 & 54,788 \\
\hline & SD & 777 & 804 & 1301 & 1048 & 1254 & 627 \\
\hline & CV & 3.15 & 2.01 & 2.59 & 1.99 & 2.29 & 1.14 \\
\hline \multirow[t]{4}{*}{$1 \mathrm{~min}$} & Mean $(\mathrm{Bq} / \mathrm{mL})$ & 23,591 & 38,981 & 50,515 & 52,674 & 55,621 & 56,965 \\
\hline & SD & 1,339 & 2,207 & 2,064 & 687 & 741 & 340 \\
\hline & CV & 5.68 & 5.66 & 4.09 & 1.30 & 1.33 & 0.60 \\
\hline & \%Dif-index* & -4.45 & -2.62 & 0.69 & 0.03 & 1.71 & 3.97 \\
\hline \multirow[t]{4}{*}{$30 \mathrm{~s}$} & Mean (Bq/mL) & 20,859 & 39,076 & 50,331 & 54,196 & 57,462 & 59,467 \\
\hline & SD & 1,422 & 2,380 & 2,165 & 1,296 & 1,156 & 1,264 \\
\hline & $\mathrm{CV}$ & 6.82 & 6.09 & 4.30 & 2.39 & 2.01 & 2.13 \\
\hline & $\%$ Dif-index ${ }^{*}$ & -15.52 & -2.38 & 0.32 & 2.92 & 5.07 & 8.54 \\
\hline \multirow[t]{4}{*}{$15 s$} & Mean $(\mathrm{Bq} / \mathrm{mL})$ & 16,346 & 37,668 & 50,078 & 55,989 & 58,993 & 62,000 \\
\hline & SD & 2,900 & 3,210 & 4,167 & 2,930 & 3,451 & 2,054 \\
\hline & CV & 17.74 & 8.52 & 8.32 & 5.23 & 5.85 & 3.31 \\
\hline & $\%$ Dif-index & -33.79 & -5.90 & -0.18 & 6.33 & 7.87 & 13.16 \\
\hline
\end{tabular}

*\%Dif-index $=$ percentage difference compared with 3-min acquisition. 
TABLE 3

Results of Phantom Study for an ${ }^{18} \mathrm{~F}-\mathrm{FDG}$ Concentration of $13 \mathrm{kBq} / \mathrm{mL}(n=5)$

\begin{tabular}{|c|c|c|c|c|c|c|c|}
\hline \multirow[b]{2}{*}{ Acquisition time } & \multirow[b]{2}{*}{ Parameter } & \multicolumn{6}{|c|}{ Sphere size (mm) } \\
\hline & & 10 & 13 & 17 & 22 & 28 & 37 \\
\hline $15 \min$ & & 6,060 & 10,662 & 11,873 & 12,923 & 12,715 & 13,041 \\
\hline \multirow[t]{3}{*}{$3 \mathrm{~min}$} & Mean $(\mathrm{Bq} / \mathrm{mL})$ & 5,570 & 10,477 & 12,019 & 13,049 & 13,275 & 13,887 \\
\hline & SD & 144 & 402 & 398 & 320 & 312 & 289 \\
\hline & CV & 2.58 & 3.84 & 3.31 & 2.45 & 2.35 & 2.08 \\
\hline \multirow[t]{4}{*}{$1 \mathrm{~min}$} & Mean $(\mathrm{Bq} / \mathrm{mL})$ & 4,510 & 9,855 & 11,705 & 13,174 & 13,993 & 14,857 \\
\hline & SD & 889 & 512 & 474 & 1048 & 80 & 428 \\
\hline & CV & 19.71 & 5.20 & 4.05 & 7.96 & 0.57 & 2.88 \\
\hline & \%Dif-index ${ }^{\star}$ & -19.03 & -5.94 & -2.61 & 0.95 & 5.41 & 6.99 \\
\hline \multirow[t]{4}{*}{$30 \mathrm{~s}$} & Mean (Bq/mL) & t & 9,356 & 11,947 & 13,133 & 14,901 & 15,822 \\
\hline & SD & & 1,233 & 990 & 1,101 & 695 & 475 \\
\hline & $\mathrm{CV}$ & & 13.18 & 8.29 & 8.39 & 4.67 & 3.00 \\
\hline & $\%$ Dif-index ${ }^{*}$ & & -10.70 & -0.60 & 0.64 & 12.25 & 13.93 \\
\hline \multirow[t]{4}{*}{$15 \mathrm{~s}$} & Mean (Bq/mL) & $t$ & 6,670 & 10,507 & 13,317 & 16,593 & 17,737 \\
\hline & SD & & 609 & 692 & 953 & 1,301 & 621 \\
\hline & CV & & 9.13 & 6.58 & 7.16 & 7.84 & 3.50 \\
\hline & $\%$ Dif-index ${ }^{\star}$ & & -36.33 & -12.58 & 2.05 & 25.00 & 27.72 \\
\hline
\end{tabular}

*\%Dif-index $=$ percentage difference compared with 3-min acquisition.

${ }^{\dagger}$ Difficult to distinguish from background noise.

\section{DISCUSSION}

The $\mathrm{BH}$ technique in $\mathrm{CT}$ has been widely investigated for radiotherapy in treatment of the lungs $(20,21)$, and deepinspiration $\mathrm{BH}$ PET/CT has recently been reported to be feasible in the clinical setting $(17,18)$. Because the specifications of the PET/CT system at our institute do not correspond to those of a recently reported protocol $(17,18)$, we focused on the single-BH technique during PET emission to enable a reduction in examination time and to optimize image fusion and SUV analysis. We propose a $\mathrm{BH}$ $\mathrm{PET} / \mathrm{CT}$ protocol in which the patient is instructed to hold his or her breath in the maximal inspiratory position during the initial scout scan, for $10 \mathrm{~s}$ during the CT scan, and during the PET scan for as long as possible. We used a respiratory monitoring device for this study. The advantage of using this system was the ability to monitor the patients' actual breathing conditions and to determine the time from the beginning to the end of motionless breath-holding.

TABLE 4

Results of Phantom Study for an ${ }^{18} \mathrm{~F}-\mathrm{FDG}$ Concentration of $7 \mathrm{kBq} / \mathrm{mL}(n=5)$

\begin{tabular}{|c|c|c|c|c|c|c|c|}
\hline \multirow[b]{2}{*}{ Acquisition time } & \multirow[b]{2}{*}{ Parameter } & \multicolumn{6}{|c|}{ Sphere size (mm) } \\
\hline & & 10 & 13 & 17 & 22 & 28 & 37 \\
\hline $15 \mathrm{~min}$ & & $2,881.29$ & $4,896.79$ & $5,548.12$ & $6,111.37$ & $6,370.27$ & $6,500.82$ \\
\hline \multirow[t]{3}{*}{$3 \mathrm{~min}$} & Mean (Bq/mL) & $\dagger$ & 4,670 & 5,504 & 6,297 & 6,640 & 7,352 \\
\hline & SD & & 155 & 257 & 448 & 174 & 273 \\
\hline & CV & & 3.32 & 4.66 & 7.12 & 2.62 & 3.71 \\
\hline \multirow[t]{4}{*}{$1 \min$} & Mean $(\mathrm{Bq} / \mathrm{mL})$ & $\dagger$ & 4,517 & 5,547 & 6,602 & 7,282 & 8,240 \\
\hline & SD & & 428 & 211 & 214 & 704 & 530 \\
\hline & CV & & 9.48 & 3.81 & 3.25 & 9.67 & 6.44 \\
\hline & $\%$ Dif-index ${ }^{*}$ & & -3.29 & 0.78 & 4.85 & 9.67 & 12.07 \\
\hline \multirow[t]{4}{*}{$30 \mathrm{~s}$} & Mean $(\mathrm{Bq} / \mathrm{mL})$ & $\dagger$ & 2,501 & 4,986 & 6,792 & 8,486 & 9,469 \\
\hline & SD & & 745 & 761 & 683 & 1,232 & 426 \\
\hline & CV & & 29.78 & 15.26 & 10.06 & 14.52 & 4.50 \\
\hline & $\%$ Dif-index ${ }^{\star}$ & & -46.45 & -9.43 & 7.86 & 27.80 & 28.79 \\
\hline \multirow[t]{4}{*}{$15 \mathrm{~s}$} & Mean (Bq/mL) & $\dagger$ & $t$ & 4,384 & 5,544 & 7,327 & 9,275 \\
\hline & SD & & & 630 & 709 & 1,208 & 868 \\
\hline & CV & & & 14.36 & 12.79 & 16.49 & 9.36 \\
\hline & $\%$ Dif-index & & & -20.35 & -11.96 & 10.34 & 26.15 \\
\hline
\end{tabular}

*\%Dif-index $=$ percentage difference compared with 3-min acquisition.

${ }^{\dagger}$ Difficult to distinguish from background noise. 
TABLE 5

Differences Between Upper Lung Area and Lower Lung Area

\begin{tabular}{lccc}
\hline \multicolumn{1}{c}{ Characteristic } & $\begin{array}{c}\text { Upper lung } \\
\text { area }(n=78)^{\star}\end{array}$ & $\begin{array}{c}\text { Lower lung } \\
\text { area }(n=30)^{\dagger}\end{array}$ & $P$ \\
\hline Patient age & $66.5 \pm 9.8$ & $66.9 \pm 9.9$ & NS \\
BMI & $22.2 \pm 3.1$ & $21.7 \pm 2.5$ & NS \\
$\begin{array}{l}\text { 18F-FDG dosage } \\
\text { (MBq/kg) }\end{array}$ & $4.66 \pm 1.48$ & $4.60 \pm 1.45$ & NS \\
BH time (s) & $56.1 \pm 22.2$ & $49.3 \pm 15.7$ & NS \\
$\begin{array}{l}\text { Maximum } \\
\text { diameter (cm) }\end{array}$ & $2.61 \pm 0.75$ & $2.28 \pm 0.63$ & 0.0410 \\
SUVmaxFB & $9.12 \pm 5.48$ & $5.32 \pm 2.74$ & 0.0003 \\
SUVmaxBH & $10.52 \pm 6.19$ & $7.85 \pm 3.91$ & NS \\
\%BH-index & $16.9 \pm 25.6$ & $51.8 \pm 49.5$ & $<0.0001$
\end{tabular}

*Upper lung area includes S1, S2, S1 + 2, S3, S4, S5, S4 + 5, and S6.

${ }^{\dagger}$ Lower lung area includes S7, S8, S9, and S10.

NS $=$ not statistically significant $(P>0.05)$.

Data are mean \pm SD.

Some patients were unable to produce motionless inspiratory action as instructed because of misunderstanding, strain, laziness, sleepiness, or confusion. We were immediately alerted to breath-holding problems by observing the real-time display of the respiratory monitoring device. This system also enabled us to exclude patients whose data were inadequate for such reasons as having a short BH (lasting fewer than $29 \mathrm{~s}$ ), being uncooperative, coughing, yawning, speaking, being deaf, and having dementia. The additional time patients are required to spend in the scanner for both FB PET/CT and BH PET/CT was about 10 min, including breathing instruction. The postprocessing time was approximately 5 min because of parallel processing and enabled

TABLE 6

Differences Between Upper Lobe and Middle-to-Lower Lobe

\begin{tabular}{|c|c|c|c|}
\hline Characteristic & $\begin{array}{l}\text { Upper lobe } \\
(n=50)^{\star}\end{array}$ & $\begin{array}{l}\text { Middle-to-lower } \\
\text { lobe }(n=58)^{\dagger}\end{array}$ & $P$ \\
\hline Patient age & $66.9 \pm 10.0$ & $66.4 \pm 9.7$ & NS \\
\hline $\mathrm{BMI}$ & $22.3 \pm 3.2$ & $21.9 \pm 2.8$ & NS \\
\hline $\begin{array}{l}{ }^{18} \mathrm{~F}-\mathrm{FDG} \text { dosage } \\
(\mathrm{MBq} / \mathrm{kg})\end{array}$ & $4.67 \pm 1.47$ & $4.61 \pm 1.47$ & NS \\
\hline $\mathrm{BH}$ time (s) & $59.5 \pm 25.0$ & $49.7 \pm 15.1$ & NS \\
\hline $\begin{array}{l}\text { Maximum } \\
\quad \text { diameter }(\mathrm{cm})\end{array}$ & $2.56 \pm 0.78$ & $2.48 \pm 0.69$ & NS \\
\hline SUVmaxFB & $8.38 \pm 5.15$ & $7.79 \pm 5.18$ & NS \\
\hline SUVmaxBH & $9.28 \pm 5.56$ & $10.21 \pm 5.93$ & NS \\
\hline$\% \mathrm{BH}$-index & $11.4 \pm 13.9$ & $39.6 \pm 45.3$ & $<0.0001$ \\
\hline $\begin{array}{l}{ }^{*} \text { Upper lobe area } \\
{ }^{\dagger} \text { Middle-to-lower I } \\
\text { S9, and S10. } \\
\text { NS = not statistic } \\
\text { Data are mean } \pm\end{array}$ & $\begin{array}{l}\text { ncludes S1, S } \\
\text { be area inclu } \\
\text { ally significant } \\
\text { SD. }\end{array}$ & $\begin{array}{l}\mathrm{S} 1+2, \text { and } \mathrm{S} 3 \\
\text { les } \mathrm{S} 4, \mathrm{~S} 5, \mathrm{~S} 4+5 \\
(P>0.05)\end{array}$ & , S6, S7, S8, \\
\hline
\end{tabular}

TABLE 7

Differences Between High-\%BH-Index Group and Low$\% \mathrm{BH}-$ Index Group

\begin{tabular}{|c|c|c|c|}
\hline Characteristic & $\begin{array}{l}\text { High \%BH-index } \\
\text { group* }^{*}(n=24)\end{array}$ & $\begin{array}{l}\text { Low } \% \text { BH-index } \\
\text { group }{ }^{\dagger}(n=84)\end{array}$ & $P$ \\
\hline Patient age & $69.7 \pm 6.1$ & $65.7 \pm 10.4$ & NS \\
\hline BMI & $22.5 \pm 3.0$ & $22.0 \pm 3.0$ & NS \\
\hline $\begin{array}{c}{ }^{18} \mathrm{~F}-\mathrm{FDG} \\
\text { dosage } \\
(\mathrm{MBq} / \mathrm{kg})\end{array}$ & $4.66 \pm 1.43$ & $4.63 \pm 1.48$ & NS \\
\hline $\mathrm{BH}$ time (s) & $51.5 \pm 17.6$ & $55.0 \pm 21.7$ & NS \\
\hline SUVmaxFB & $5.39 \pm 2.22$ & $8.83 \pm 5.50$ & 0.0076 \\
\hline SUVmaxBH & $9.51 \pm 3.79$ & $9.86 \pm 6.22$ & NS \\
\hline Volume $\left(\mathrm{cm}^{3}\right)$ & $7.79 \pm 6.13$ & $16.33 \pm 13.01$ & 0.0019 \\
\hline $\begin{array}{l}\text { Maximum } \\
\quad \text { diameter }(\mathrm{cm})\end{array}$ & $2.13 \pm 0.57$ & $2.63 \pm 0.74$ & 0.0032 \\
\hline
\end{tabular}

${ }^{*} \mathrm{High}-\% \mathrm{BH}-$-index group: higher than 37.

${ }^{\dagger}$ Low-\%BH-index group: lower than 37 .

NS $=$ not statistically significant $(P>0.05)$.

Data are mean \pm SD.

the scanner to examine the next patient. The advantages of this proposed protocol were its short acquisition time, high probability of technical success in completing the study in cooperative patients, and simple postprocessing. For radiation dose, the additional effective dose of BH CT was about $15 \%$ of whole-body PET/CT, which is considered acceptable for routine clinical examinations.

Our clinical study addressed the \%BH-index of consecutive patients with various lung tumors in the entire lung fields bilaterally. The \%BH-index showed an unavoidable scatter of values; thus, the \%BH-index might have been affected by the following factors: $\mathrm{BH}$ time, lesion size, lesion location, ${ }^{18} \mathrm{~F}-$ FDG dosage, patient age, patient sex, and BMI, among others. We observed a significantly high \%BH-index in the lower lung

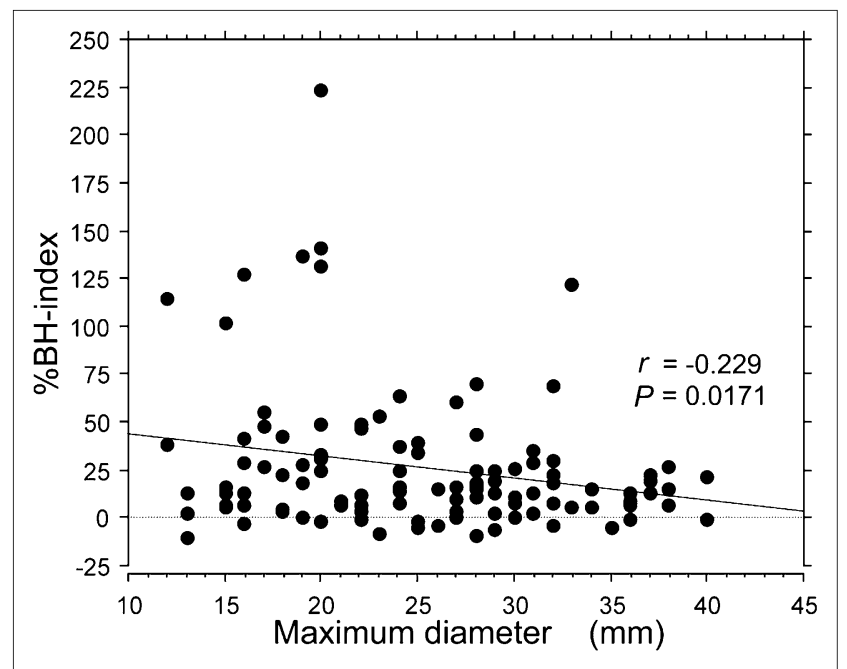

FIGURE 3. Graph of regression analysis between \%BH-index and maximum lesion diameter. Weak inverse relationship was observed. 


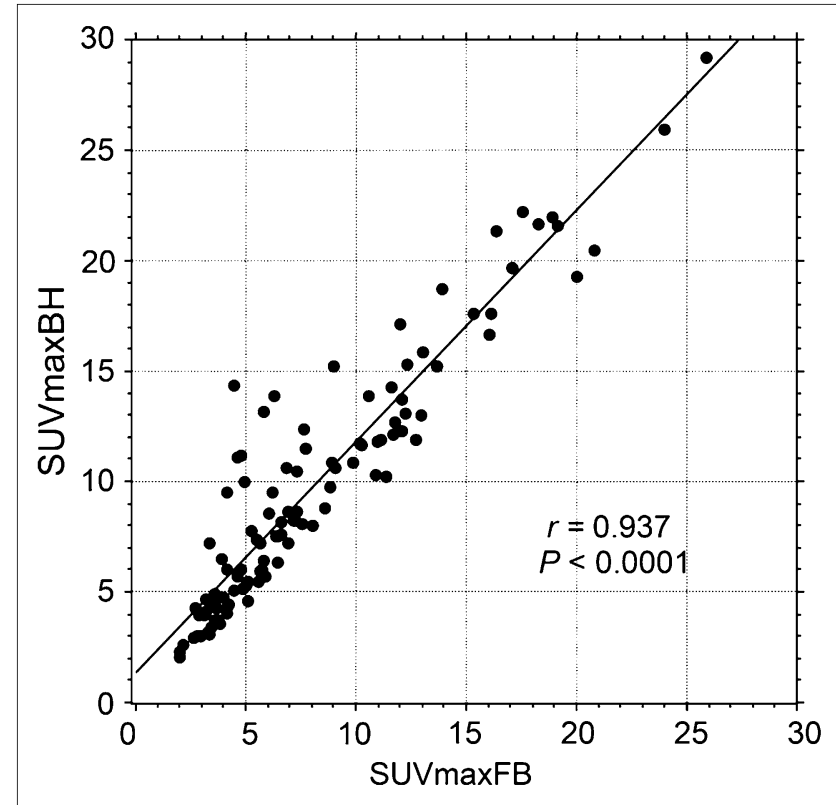

FIGURE 4. Graph of regression analysis between SUVmaxFB and SUVmaxBH. Significant relationship was observed.

area and middle-to-lower lobe (Tables 5 and 6). This result indicates that the movement of the diaphragm synchronously influences pulmonary movement during the breathing cycle; the depth, cycle speed, and waveform of respiratory motion are the factors that lead to a reduction in the SUVmax of FB PET and cause blurring of the PET images.

Although the formula for lesion volume used in this study did not give an accurate volume, the formula was applied as a simple alternative for estimating lesion volume. In the pre- sent study, lesion volume and maximum diameter in the high-\%BH-index group were significantly lower than those in the low-\%BH-index group (Table 7). In FB PET images, the area of ${ }^{18} \mathrm{~F}-\mathrm{FDG}$ accumulation tended to exceed the contour of the lesion on CT images, indicating blurred ${ }^{18} \mathrm{~F}$ FDG accumulation around the lung tumor, especially in small tumors (Fig. 5). However, large lesions do not always show ${ }^{18}$ F-FDG blurring around the lung tumor. This phenomenon may rely on the uniformity of ${ }^{18}$ F-FDG accumulation, especially in large tumors, because of factors such as hypoxia, necrosis, and cavitation (Fig. 6). Throughout the duration of the breathing cycle, within areas of heterogeneous ${ }^{18} \mathrm{~F}$-FDG accumulation each pixel with a maximum ${ }^{18} \mathrm{~F}-\mathrm{FDG}$ accumulation is averaged while moving in a complicated manner. The lesion size, shape, and nonuniform distribution of ${ }^{18}$ F-FDG activity within the tumor are also factors that reduce the SUVmax of FB PET, particularly in large tumors.

Theoretically, BH PET/CT achieves ideal image fusion; however, clinically, several patients showed misregistration in fusion PET/CT images. The extent of lung inspiration levels differs between BH CT and BH PET. To avoid this misregistration in fusion PET/CT images, it is important that patients hold their breath at the same level of inspiration. A previous report documented that SUV measures are affected by the degree of mismatch between CT and PET datasets (3). In our SUVmax analysis, we eliminated patients with obvious mismatch. As a result, because the depth of inspiration was measured using a respiratory monitoring device, almost all patients showed a perfect match between $\mathrm{BH} \mathrm{CT}$ and $\mathrm{BH}$ PET. However, the mismatch between FB CT and FB PET was disregarded because FB PET/CT was the clinically used standard protocol.

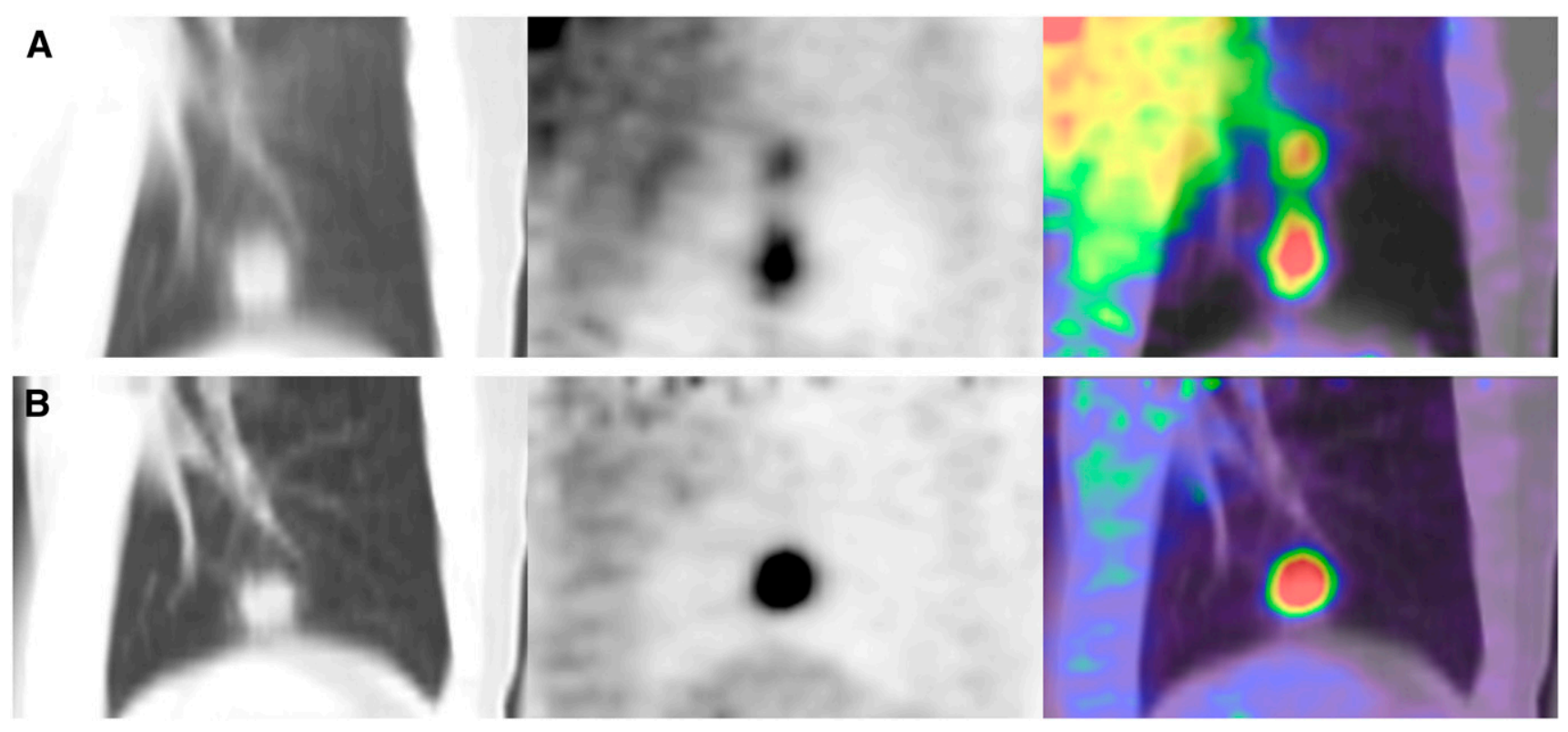

FIGURE 5. Man (63 y) with lung cancer in left lower lobe: coronal FB PET/CT (A) and coronal BH PET/CT (B). Maximum lesion diameter was $20 \mathrm{~mm}$ and BH time was $95 \mathrm{~s}$. SUVmax for FB PET and BH PET was 4.44 and 14.35, respectively. \%BH-index was 223.2. Blurring of ${ }^{18} \mathrm{~F}-\mathrm{FDG}$ accumulation was more apparent in fusion FB PET/CT than in fusion BH PET/CT. Complete superimposition was noted in fusion BH PET/CT. 

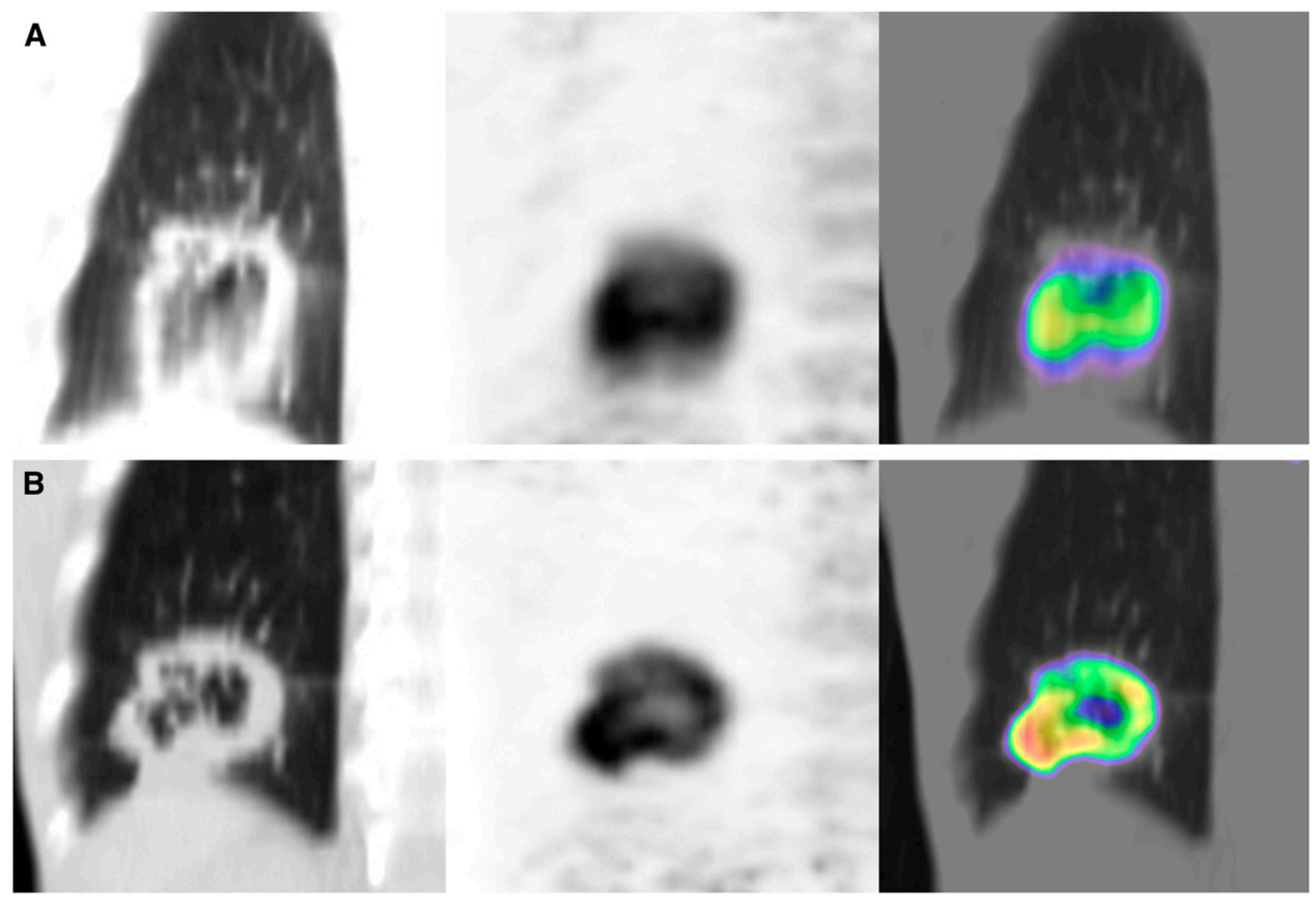

FIGURE 6. Man (70 y) with lung cancer in right lower lobe: coronal FB PET/CT (A) and coronal BH PET/CT (B). Maximum lesion diameter was $58 \mathrm{~mm}$, with cavitation; BH time was $51 \mathrm{~s}$. SUVmax for FB PET and BH PET was 16.71 and 18.84, respectively. $\% \mathrm{BH}$-index was 12.7. Respiratory motion artifact was noted in FB CT. Marginal ${ }^{18} \mathrm{~F}-\mathrm{FDG}$ accumulation in lung lesion with cavitation was clearly visualized on fusion BH PET/CT.

The present study has limitations. BH PET was performed during a short acquisition time; a PET acquisition time of $1 \mathrm{~min} /$ bed position was previously reported (19), but an acquisition time of less than $1 \mathrm{~min}$ has not been reported in the clinical setting. In our study, the BH time was equivalent to the acquisition time of $\mathrm{BH}$ dynamic PET. As both images were reconstructed using the same algorithm, the BH PET images were low-count images compared with the FB PET images acquired in 3 min (Figs. 5 and 6). Strictly speaking, SUVmax comparisons between short and long acquisition times are impossible because SUVmax is inherently influenced by noise, and the SUVmax of a short acquisition is affected by noise to a greater degree than that of a 3-min acquisition. To the best of our knowledge, no study has been conducted that compares the SUVmax of shorter acquisition times with that of longer acquisition times using the same type of PET/CT as the one at our institution. To address this gap in the literature, we performed a phantom study analyzing the features of visual detectability and the tendency of $\%$ Dif-index (Tables 2-4). Cases of shorter acquisition time and lower ${ }^{18} \mathrm{~F}$-FDG concentration were insufficient for SUVmax analysis; therefore, in the clinical study we excluded patients with short breath-holding and those in whom pulmonary lesions showed lower ${ }^{18} \mathrm{~F}-\mathrm{FDG}$ accumulations on whole-body PET. Clinically, all pulmonary tumors of participating patients were easily distinguished from the pulmonary background activity, and ROIs were easily placed around the pulmonary tumors in BH PET. The target pulmonary lesion appeared to have high signal-to-noise ratio on $\mathrm{BH}$ PET; this could be because of the reduced background activity of the expanded lung in deep inspiration. A previous article has also commented on the increased target-to-background ratio in the thorax (17).

In the phantom study, the \%Dif-index showed a tendency to be higher in large spheres and lower in small spheres. The \%BH-index in the clinical study comprises the features of $\%$ Dif-index in the phantom study; however, the tendency in the clinical study of the \%BH-index to be higher in small pulmonary lesions conflicts with the results of the phantom study. This conflict also supports the hypothesis that respiratory motion affects the \%BH-index more strongly than it affects the \%Difindex of the phantom study, especially in small lesions.

A weak inverse relationship between patient age and $\mathrm{BH}$ time indicates that older patients tend to hold their breath for a shorter period than do younger patients. Respiratory function may influence the $\mathrm{BH}$ time. 
In addition, the mean radiation effective dose of FB CT was slightly higher than that of BH CT. The differences in expanded lung density between FB and deep-inspiration $\mathrm{BH}$ may have influenced the auto tube current modulation.

Previous reports show that ${ }^{18} \mathrm{~F}-\mathrm{FDG}$ dosage and BMI affect the quality of PET images on LSO-based PET (2224); however, in our results the factors of ${ }^{18} \mathrm{~F}-\mathrm{FDG}$ dosage, $\mathrm{BMI}$, and $\mathrm{BH}$ time were not associated with \% BH-index.

To reflect the features of phantom data, we restricted the maximum diameter of lung tumor in the clinical study to a range of 10-40 $\mathrm{mm}$; however, the unexposed results, including large lesions more than $40 \mathrm{~mm}$ in diameter, showed the same tendencies as those described earlier and statistical differences (not shown).

The proposed BH PET/CT technique may be one of the feasible methods for SUVmax analysis and tumor registration in clinical PET/CT and is expected to enable precise SUVmax to be obtained in lung cancer. Determining a precise SUVmax can contribute to the reliability of chemoradiation therapy monitoring because BH PET/CT with breathing information provides similar scanning conditions from the baseline scan to follow-up scans. Future advances in detector and acquisition technology will reduce acquisition time and make this proposed $\mathrm{BH}$ technique clinically practical for whole-body PET/CT.

\section{CONCLUSION}

We described a phantom data analysis and SUVmax analysis of BH PET/CT data in 108 cases of lung cancer. SUVmax of BH PET showed an increase in the lower lung area. Lesions in the high \% BH-index group appeared small compared with those in the low $\% \mathrm{BH}$-index group. The SUVmax of FB PET should not be accepted as accurate, especially in the lower lung area and for small pulmonary lesions. BH PET/CT realizes a complete match between CT and PET, with the expectation of obtaining precise SUVmax, and is thus recommended as part of the standard protocol for lung cancer.

\section{REFERENCES}

1. Goerres GW, Kamel E, Heidelberg TN, Schwitter MR, Burger C, von Schulthess GK. PET-CT image coregistration in the thorax: influence of respiration. Eur $J$ Nucl Med Mol Imaging. 2002;29:351-360.

2. Beyer T, Antoch G, Blodgett T, Freudenberg LF, Akhurst T, Mueller S. Dualmodality PET/CT imaging: the effect of respiratory motion on combined image quality in clinical oncology. Eur J Nucl Med Mol Imaging. 2003;30:588-596.
3. Erdi YE, Nehmeh SA, Pan T, et al. The CT motion quantitation of lung lesions and its impact on PET-measured SUVs. J Nucl Med. 2004;45:1287-1292.

4. Pan T, Mawlawi O, Nehmeh SA, et al. Attenuation correction of PET images with respiration-averaged CT images in PET/CT. J Nucl Med. 2005;46:1481-1487.

5. Beyer T, Rosenbaum S, Veit $\mathrm{P}$, et al. Respiration artifacts in whole-body ${ }^{18} \mathrm{~F}$ FDG PET/CT studies with combined PET/CT tomographs employing spiral CT technology with 1 to 16 detector rows. Eur J Nucl Med Mol Imaging. 2005; 32:1429-1439.

6. Gilman MD, Fischman AJ, Krishnasetty V, Halpern EF, Aquino SL. Optimal CT breathing protocol for combined thoracic PET/CT. AJR. 2006;187:1357-1360.

7. Allen-Auerbach M, Yeom K, Park J, Phelps M, Czernin J. Standard PET/CT of the chest during shallow breathing is inadequate for comprehensive staging of lung cancer. J Nucl Med. 2006;47:298-301.

8. Juergens KU, Weckesser M, Stegger L, et al. Tumor staging using whole-body high-resolution 16-channel PET-CT: does additional low-dose chest CT in inspiration improve the detection of solitary pulmonary nodules? Eur Radiol. 2006;16:1131-1137.

9. Aquino SL, Kuester LB, Muse VV, Halpern EF, Fischman AJ. Accuracy of transmission CT and FDG-PET in the detection of small pulmonary nodules with integrated PET/CT. Eur J Nucl Med Mol Imaging. 2006;33:692-696.

10. Nehmeh SA, Erdi YE, Ling CC, et al. Effect of respiratory gating on reducing lung motion artifacts in PET imaging of lung cancer. Med Phys. 2002;29:366-371.

11. Dewan NA, Gupta NC, Redepenning LS, Phalen JJ, Frick MP. Diagnostic efficacy of PET-FDG imaging in solitary pulmonary nodules: potential role in evaluation and management. Chest. 1993;104:997-1002.

12. Nestle U, Kremp S, Schaefer-Schuler A, et al. Comparison of different methods for delineation of ${ }^{18} \mathrm{~F}$-FDG PET-positive tissue for target volume definition in radiotherapy of patients with non-small cell lung cancer. J Nucl Med. 2005;46:1342-1348.

13. Biehl KJ, Kong FM, Dehdashti F, et al. ${ }^{18}$ F-FDG PET definition of gross tumor volume for radiotherapy of non-small cell lung cancer: is a single standardized uptake value threshold approach appropriate? J Nucl Med. 2006;47:1808-1812.

14. Nehmeh SA, Erdi YE, Ling CC, et al. Effect of respiratory gating on quantifying PET images of lung cancer. J Nucl Med. 2002;43:876-881.

15. Nehmeh SA, Erdi YE, Rosenzweig KE, et al. Reduction of respiratory motion artifacts in PET imaging of lung cancer by respiratory correlated dynamic PET: methodology and comparison with respiratory gated PET. J Nucl Med. 2003;44: 1644-1648.

16. Nehmeh SA, Erdi YE, Pan T, et al. Four-dimensional (4D) PET/CT imaging of the thorax. Med Phys. 2004;31:3179-3186.

17. Nehmeh SA, Erdi YE, Meirelles GS, et al. Deep-inspiration breath-hold PET/CT of the thorax. J Nucl Med. 2007;48:22-26.

18. Meirelles GS, Erdi YE, Nehmeh SA, et al. Deep-inspiration breath-hold PET/ CT: clinical findings with a new technique for detection and characterization of thoracic lesions. J Nucl Med. 2007;48:712-719.

19. Halpern BS, Dahlbom M, Quon A, et al. Impact of patient weight and emission scan duration on PET/CT image quality and lesion detectability. J Nucl Med. 2004;45:797-801.

20. Hanley J, Debois MM, Mah D, et al. Deep inspiration breath-hold technique for lung tumors: the potential value of target immobilization and reduced lung density in dose escalation. Int J Radiat Oncol Biol Phys. 1999;45:603-611.

21. Mah D, Hanley J, Rosenzweig KE, et al. Technical aspects of the deep inspiration breath-hold technique in the treatment of thoracic cancer. Int J Radiat Oncol Biol Phys. 2000;48:1175-1185.

22. Everaert H, Vanhove C, Lahoutte T, et al. Optimal dose of ${ }^{18} \mathrm{~F}-\mathrm{FDG}$ required for whole-body PET using an LSO PET camera. Eur J Nucl Med Mol Imaging. 2003;30:1615-1619.

23. Halpern BS, Dahlbom M, Auerbach MA, et al. Optimizing imaging protocols for overweight and obese patients: a lutetium orthosilicate PET/CT study. $\mathrm{J}$ Nucl Med. 2005;46:603-607.

24. Lodge MA, Badawi RD, Gilbert R, Dibos PE, Line BR. Comparison of 2-dimensional and 3-dimensional acquisition for ${ }^{18} \mathrm{~F}$-FDG PET oncology studies performed on an LSO-based scanner. J Nucl Med. 2006;47:23-31. 